\title{
Three Dimensional Velocity Images of The North-South Zone in China with LSQR Algorithm
}

\author{
Liu Yike Chang Xu \\ Institute of Geophysics, Chinese Academy of Sciences \\ Chaoyang district Datun Road A11, Beijing, 100101, China \\ Email: ykliu@mail.c-geos.ac.cn
}

\begin{abstract}
In this paper three dimensional velocity images of the crust and upper mantle beneath the North South Zone are reconstructed using $10277 \mathrm{P}$ wave arrivals of regional earthquakes recorded at 157 stations in the study area with LSQR algorithm. The image results indicate that: 1 . There is a significant lateral heterogeneity in the crust and upper mantle beneath the North South Zone persisting down to $450 \mathrm{~km}$ in depth. 2. The velocity images of the upper crust show the features closely related to the well known tectonic features on the surface. 3 . The low velocity layer exists in a very wide range of the mid-crust. The lowest velocity value is near $5.60 \mathrm{~km} / \mathrm{s} .4$. in a long narrow piece of area between $25^{\circ} \mathrm{N}-38^{\circ} \mathrm{N}$ and $100.0^{\circ} \mathrm{E}-103.2^{\circ} \mathrm{E}$, the low velocity anomaly about $7.49 \mathrm{~km} / \mathrm{s}$ appears at the top of the upper mantle. The image result at a depth of $120 \mathrm{~km}$ also outlines dearly the welded boundaries between the different geological blocks. The statistical data show that seismic activity is related to the velocity structure of the North South Zone: almost all of the major earthquakes took place in the transition strips between high and low velocity zones in the crust above $20 \mathrm{~km}$.
\end{abstract}

Key words: The North-South Zone of China, Three Dimensional velocity image, Seismic Tomography Method, LSQR algorithm.

\section{Introduction}

In the middle of the Chinese continent exists an important geomorphological border line. In general, this shallow earthquake concentrated zone with a north-south direction in the middle region of China is known as the North-South seismic Zone, and it is also a transition zone in the tectonic structure of China. We call the region the North-South Zone $\left(22.0^{\circ} \mathrm{N}-40.0^{\circ} \mathrm{N}, 97.0^{\circ} \mathrm{E}-\right.$ $107.5^{\circ} \mathrm{E}$ ). Based on the tectonic structure sketch of China (Fig. 1) (Huang Ji-qing,1980), it is not difficult to find that the tectonic structure in the North-South Zone is very complex. At the end of the southern part and on the west side of the region are the tectonically active Kunlun- Qinling and Yunnan Tibet geosyncline fold regions. The North-South Zone is divided by these tectonic units. As shown in Figure 2, the North-South Zone is also a strong seismic active zone within the Chinese continent. The zone possesses crisscrossing faults, and earthquakes occur frequently more than ten destructive earthquakes occurred there in the past. It is easy to think that the cases mentioned above are directly relevant to the complex tectonic pattern in the North-South Zone, and it appears under the whole tectonic background of the Chinese continent, so it has certainly important impacts on the tectonization of the Chinese continent. Therefore the studying of the tectonic features in the North-South Zone is very important in understanding the tectonization of the Chinese continent. 


\section{Data}

We selected 577 earthquakes in the study region this study. In order to guarantee the accuracy of the initial seismic source locations, localized results of the earthquakes within the study region are from the local networks. The magnitude of earthquakes is usually greater than 4.0 within the study region. Some earthquakes with 3.5-4.0 are also added in the study area where distribution of stations and epicenters is rare, such as the Alxa block, but the records of each event are generally not less than 15. All data are recorded on the Gansu, Qinghai, Ningxia, Sichuan, Young and Shane networks and the Guying station. The total number of stations in 157. The error in the arrival time is generally smaller than $0.2 \mathrm{~s}$. The altitude of stations is corrected automatically. Distributions of the regional earthquake epicenters and seismic stations used for this study are in Figure 3 and Figure 4.

\section{The Initial Model}

In general, the selection of the initial model plays an important role in the inversion process, We designed various models with consideration to the research results published about the medium structure and velocity distribution of the crust and mantle beneath the North-South Seismic Zone and adjacent regions in recent years. We selected more than 10 earthquakes with MB $>5$ which occurred in the central, northern and southern parts of the North-South Zone, by simulating the rays to yield an average travel time residual usually not exceeding $\pm 2.0 \mathrm{~s}$. The final reference velocity values at various depths are shown in Table 1 . Here we assume that the velocity changes linearly form one depth to the next.

Table 1

\begin{tabular}{|l|l|l|l|l|l|l|l|l|l|}
\hline Depth $(\mathrm{km})$ & 0 & 3 & $20^{-0}$ & $20^{+0}$ & $50^{-0}$ & $50^{+0}$ & 85 & 120 & 200 \\
\hline Velocity $(\mathrm{km} / \mathrm{s})$ & 4.60 & 5.75 & 6.20 & 6.60 & 7.00 & 7.80 & 7.94 & 8.08 & 8.39 \\
\hline
\end{tabular}

\section{Linear of Inversion Problem}

We adopt a spherical coordinate system $(r, \theta, \phi)$ in this study. The volume to be studied is split into hexahedral by coordinate surfaces

$$
r_{i}=c_{i}, \quad \mathrm{i}=1,2, \cdots, \mathrm{I} ; \quad \theta_{j}=c_{j}, \mathrm{j}=1,2, \cdots, \mathrm{J} ; \phi_{k}=c_{k}, \quad \mathrm{k}=1,2, \cdots, \mathrm{K} .
$$

where $c_{i}, c_{j}, c_{k}$ are given constants. By the selection of $r_{i}^{-0}$ and $r_{i}^{+0}$ the vertical discontinuity surface at depth of $r_{i}$ is introduced. Usually, the $c_{i}, c_{j}, c_{k}$ values are taken at uneven intervals. Owing to scarce dense extent of the distribution of earthquake epicenters and stations, the specific value is determined to ensure enough rays crossing over every grid. By means of the above mentioned method, the total study region is subdivided into uneven size hexahedral. The relative slowness perturbation of a vertex of a hexahedron is taken as a parameter to be solved, and the velocity at any point within a grid is given by an interpolation function.

The formulation of inversion is to derive relationships between the data space and model space based on physical principle. The travel time $T_{i j}$ from a point source $\mathrm{i}$ to receiver $\mathrm{j}$ is given by an integration along the ray-path in an isotropic 


$$
T_{i j}=\int_{L_{i j}} u(r) d s
$$

where $u(r)$ (slowness) is reciprocal of wave velocity along the ray-path $L_{i j}, D s$ is intergrationl curve segment. According to Fermat's principle, the ray-path spreads along the minimum time direction, and the effect of perturbation on the travel time is second order. The travel time is determined by both ray-path and velocities in the medium. Therefore, the inversion problem of travel time will be highly non-linear. But the problem can be linearized for the derivation of travel time caused by the changed ray-path is second order that can be ignored(Liu, F., 1986).

The linearized equation can be given as following:

$$
\delta T_{i j}=\int_{L_{i j}} \delta u \delta s
$$

In a spherical coordinate system the differential equations of the path are as follows.

$$
\left\{\begin{array}{l}
\frac{d r}{d t}=v^{2} p_{r}, \frac{d \theta}{d t}=\frac{v^{2}}{r} p_{\theta}, \frac{d \phi}{d t}=\frac{v^{2}}{r \sin \theta} p_{\phi}, \\
\frac{d p_{r}}{d t}=\frac{v^{2}}{r}\left(p_{\theta}^{2}+p_{\phi}^{2}\right)-\frac{1}{v} \frac{\partial \nu}{\partial \vec{\partial}}, \\
\frac{d p_{\theta}}{d t}=-\frac{v^{2}}{r}\left(p_{r} p_{\theta}-\cos \theta p_{\phi}^{2}\right)-\frac{1}{r v} \frac{\partial v}{\partial \theta}, \\
\frac{d p_{\phi}}{d t}=-\frac{v^{2}}{r}\left(p_{r} p_{\phi}+\cos p_{\theta} p_{\phi}\right)-\frac{1}{r \sin \theta} \frac{d v}{\partial \phi} .
\end{array}\right.
$$

where $p=\left(p_{r,}, p_{\theta}, p_{\phi}\right)$ is the slowness vector, $p_{r}=\frac{\cos \gamma_{r}}{v}, p_{\theta}=\frac{\cos \gamma_{\theta}}{v}, p_{\phi}=\frac{\cos \gamma_{\phi}}{v}$, and $\cos \gamma_{r}, \cos \gamma_{\theta}, \cos \gamma_{\phi}$ are the direction cosine of the ray:

In equation (2), the perturbation of arrival time can be written as following. The paths of rays are composed of $\mathrm{N}$ segments, and one is $S_{l}$ 。

$$
\delta T_{i j}=\sum_{l=1}^{N} \delta t_{l}, \quad \delta t_{l}=\frac{s_{l}}{2}\left(\delta \frac{1}{v_{l-1}}+\delta \frac{1}{v_{l}}\right)
$$

Combing equation (2), (3) and (4), the perturbation of time is as following. 


$$
\delta T_{i j}=\sum_{l=1}^{N}-\frac{s_{l}}{2}\left(\frac{1}{v_{l-1}^{2}} \sum_{\substack{m=i \\ n=j \\ q=k}}^{j+1} \frac{\partial v_{l-1}}{\partial+1} \delta v^{m, n, p} \frac{1}{v_{l}^{2}} \sum_{\substack{m=i \\ n=j \\ m=k \\ q=k}}^{\substack{k+1 \\ j+1}} \frac{\partial v_{l}}{\partial v^{m, n, p}} \delta v^{m, n, p}\right.
$$

Because of source and receiver distribution and linearized for inversion problem, there exist illposed problem when finding equation. There may be inconsistencies in the Equations or more parameters than equations. Equation (5) may be not have solution, or no unique solution. Some solutions may be pseudoimages(Chang Xu, 1996). If condition number is too big, the process to find equation (5) is not stabilitive which will cause algorithm unstability. Therefore, choosing a suitable algorithm for a ill-posed problems is an important presupposition for deriving a true solution.

\section{The LSQR Algorithm of Rreconstructed Velocity Images}

Paige (Paige, C.C.,1982) proposed LSQR method to deal with unsymmetric matrix and least squares problems. It takes less computer time and a few memory space, and is stability for illpose problem like equation (5).

LSQR is summarized as:

1)Initial condition

$$
\begin{aligned}
& \beta_{1} u_{1}=b, \alpha_{1} v_{1}=A^{T} u_{1}, \mathrm{w}_{1}=v_{1}, \mathrm{X}_{0}=0, \mathrm{~d}_{1}=0 \\
& \gamma_{1}=0, \gamma_{1}=0, \varphi_{1}=\beta_{1}, \rho=\alpha_{1}
\end{aligned}
$$

where $b_{1}, u_{1}$ are $m$ dimension vectors, $d_{1}, v_{1}, w_{1}, x_{0}$ are $n$ dimension vectors, $\beta_{1}, \alpha_{1}, \gamma_{1}, \varphi_{1}, \rho_{1}$ are real values.

2) $Q R$-factorization

$$
\begin{aligned}
& \gamma_{i+1} u_{i+1}=A v_{i+1}-\alpha_{i} u_{i} \\
& \gamma_{i+1} d_{i+1}=\lambda v_{i}-\alpha_{i} d_{i} \\
& \beta_{i+1}=\left(\gamma_{i+1}^{2}+\gamma_{i+1}^{2}\right)^{1 / 2} \\
& u_{i+1}=\gamma_{i+1} u_{i+1} / \beta_{i+1} \\
& d_{i+1}=\gamma_{i+1} d_{i+1} / \beta_{i+1} \\
& v_{i+1}=\lambda d_{i+1}-\beta_{i+1} v_{i} \\
& \alpha_{i+1} v_{i+1}=A^{T} u_{i+1}-v_{i+1}
\end{aligned}
$$


3) Parameters correction

4) Iteration solution

$$
\begin{aligned}
& \rho_{i}=\left(\rho_{i}^{2}+\beta_{i+1}^{2}\right)^{1 / 2} \\
& c_{i}=\rho_{i} / \rho_{i} \\
& s_{i}=\beta_{i+1} \rho_{i} \\
& \theta_{i+1}=s_{i+1} \alpha_{i+1} \\
& \rho_{i+1}=-c d_{i+1} \\
& \varphi_{i}=c_{i} \varphi_{i} \\
& \varphi_{i+1}=s_{i} \varphi_{i}
\end{aligned}
$$

$$
\begin{aligned}
& x_{i}=x_{i-1}+\left(\varphi_{i} / \rho_{i}\right) w_{i} \\
& w_{i+1}=v_{i+1}-\left(\theta_{i+1} / \rho_{i}\right) w_{i}
\end{aligned}
$$

where i denotes iterative variable.

\section{Three Dimensional Velocity Images of The North-South Zone Of China}

Figure 5 is the velocity image at a depth of $3 \mathrm{~km}$. It is the same as the velocity image at a depth of $20^{-0} \mathrm{~km}$. In fact, it reflects lateral heterogeneity of the velocity in the upper crust (above $20 \mathrm{~km}$ ). In Figure $5,6,7,8$, the red color represents low velocity zones, while the blue indicates high velocity zones, as shown is the color symbol. From Figure 5 it can be seen that the velocity distribution is closely correlated to the tectonic features on the surface. The most attractive low velocity zone is in the Sichuan basin. In its north part the red strip corresponds to the Yinchuan basin in the Ordos marginal fold belt platform. Within Yunnan Province, there are two red regions from southwestern Kunming to Xishuangbanna. They are two low velocity regions cut apart by a related high velocity region corresponding to the Ailao Mountains. The conspicuous high velocity strip on the west side and southern spread of the Sichuan basin corresponds to the Kang-Dian geoaxis which runs form the Ailao Mountains the south through Yuanmo, Dukou, Xichang and Miannin to Kangding where it runs north spreading into separate two branches. The northwest one is at the east foot of the Animaqing Mountains; the center of the northeastern one is near Zoige: then toward the northwest the high velocity region in west of Yinchuan basin is the Urad uplift platform. Comparing Figure 6 with Figure 5, it is not difficult to find almost all of the major earthquakes distributed on the transition zones between high and low velocity regions.

Figure 6 is the velocity image at a depth of $20^{+0} \mathrm{~km}$. It represents the velocity distribution in the mid-crust of this region. As shown in Figure 6, form the velocity value of the parametrial model a large-scale negative abnormality took place in the region. The maximum negative perturbation is $-15 \%$. Corresponding to the reference velocity of $6.60 \mathrm{~km} / \mathrm{s}$. the minimum velocity reaches $5.60 \mathrm{~km} / \mathrm{s}$ in the low velocity zone. 
By projected mid-strong shallow source earthquake locations which are over $15 \mathrm{~km}$ depth on the velocity image of a depth $20^{+0} \mathrm{~km}$, it can be statistically found that the most of the earthquakes are distributed within the low regions and on their boundaries.

Figure 7 is the velocity image at a depth of $50 \mathrm{~km}$. It shows the velocity distribution at the top of the upper mantle. From the figure it can be seen that in an elongated area which is form $25^{\circ}$ $\mathrm{N}$ to $38^{\circ} \mathrm{N}$ in latitude and form $100.0^{\circ} \mathrm{E}$ to $103.2^{\circ} \mathrm{E}$ in longitude, the $\mathrm{P}$ wave velocity appears as a negative abnormal. The perturbation is generally less than- $4 \%$ of the initial value of $7.80 \mathrm{~km} / \mathrm{s}$. So the velocity is $7.49 \mathrm{~km} / \mathrm{s}$ in the abnormal area. In the high velocity areas which are the east area of $103.2^{\circ} \mathrm{E}$ and the south area of $25^{\circ} \mathrm{N}$, the perturbation is generally greater than $+4 \%$ of the reference velocity of $7.80 \mathrm{~km} / \mathrm{s}$, and the velocity value is greater than $8.10 \mathrm{~km} / \mathrm{s}$.

The velocity abnormality in this depth is undoubtedly the result of complex structure movement; therefore, two possibilities can be inferred. The first one is that the low velocity abnormality is explained as the abnormal upper mantle appearing in this area, which possibly represents a local melt in this part of the upper mantle. The another one is the undulation of the crust thickness. From the latter deduction, as shown in Figure 7, the crust outline in the study region from the Qilian Mountains through Yongdeng and Lanzhou to Tianshui and Wudu toward the southwest to Xichang is the incline zone of the $M$ interface toward the east protrude. The east is thin and the west is thick. From Xichang toward the south, the equi-depth line of M interface protrude in a tongue form to the east and the west. .

It is worth pointing out that in Figure 7 there are two particularly abnormal regions. One of them is in the Zoige high velocity zone; another one is in the Tengchong low velocity zone. Based on previous results, the crust thickness of the former is greater than $54 \mathrm{~km}$, and the latter one is less than $40 \mathrm{~km}$. The velocity abnormality in both regions, of course, cannot be explained with the undulation of $\mathrm{M}$ interface. For the Zoige zone, from Figure 5, and 7, we see that their velocity values are all relatively high over the equi-depth surfaces. This implies that there is a high velocity block in the Zoige region. This result is useful for determining the existence of the Zoige covering block geologically. For the Tengchong region, from $20^{+0} \mathrm{~km}$ down to $120 \mathrm{~km}$, a low velocity column for ms. There are also 10 high temperature hot springs accompanying the high seismicity. It is a tectonic active region with characteristics of the "intergrowth" development of three geological phenomena: volcanoes, earthquakes and hot springs.

Figure 8 is the velocity image at a depth of $120 \mathrm{~km}$. It not only reveals the existence of striping velocity differences between the relatively stable Yangtze paraplatform and relatively active fold belts on the west side, but also clearly outlines the welded boundaries among the blocks. Along the west side a series of marked low velocity regions on the welded boundaries is all combined zones of different tectonic elements. They are respectively the adhesive places of the Qinling fold system and the Songpan-Ganzi fold system with Yangtze paraplatform. The low velocity area to west is a combined zone of the Songpan-Ganzi fold system and the Sanjiang fold system with the Yangtze paraplatform. The low velocity area in the south at $25^{\circ} \mathrm{N}$ and east 102.5 - $\mathrm{E}$ is the consolidation belt belonging to the Yangtze paraplatform with Huanan block along Nanpanjiang. The northwest direction's low velocity zone form Yibin to west Chengdu within the Yangtze paraplatform cuts apart two high velocity zones. The northeast one is the upper Yangtze platform fold belt, and the southwest one is the Kang-Dian geoaxis. This low velocity zone seems to be taken as the consolidation boundary between the above mentioned two blocks within the Yangtze paraplatform. 


\section{Conclusion}

In this paper three dimensional velocity images of the crust and upper mantle beneath the North-South Zone are obtained by using the seismic tomography method with LSQR algorithm. Image results indicate that: a)there is striking lateral heterogeneity in the crust and upper mantle beneath the North-South Zone, persisting down to $450 \mathrm{~km}$ in depth; b)the velocity images of the upper crust show features closely related to the well known tectonic features on the surface: the velocity under Sichuan Basin is apparently low and along the Kang-Dian geoaxis is obviously high; c)the low velocity layer exists within a very wide region of the mid crust, and the low velocity value is near $5.60 \mathrm{~km} / \mathrm{s}$; d) in a strip area between $25^{\circ} \mathrm{N}-38^{\circ} \mathrm{N}$ and $100.0^{\circ} \mathrm{E}-103.2^{\circ} \mathrm{E}$, the low velocity anomaly with the value $7.49 \mathrm{~km} / \mathrm{s}$ appears at the top of the upper mantle. It is possibly the result of local melt mantle body surging up due to the tectonic activity as well as the crust getting thin.

\section{References}

Chang, X., 1996.Relationship between ray distribution and reconstructed velocity image in reflection tomography. J. Applied Geophysics,35:145-150.

Huang Ji-qing, 1980. Tectonic stucture and Evolution of China, sciences Press (in Chinese).

Liu, F., 1986. On the Tomographic inverse method used in velocity image reconstruction. Chinese J. Geophysics, 32: 62-80.

Paige, C.C., and Saunders, M.A., 1982. LSQR: Sparse linear equations and least squares problems, AMC Transactions. Math., 8:195-209.

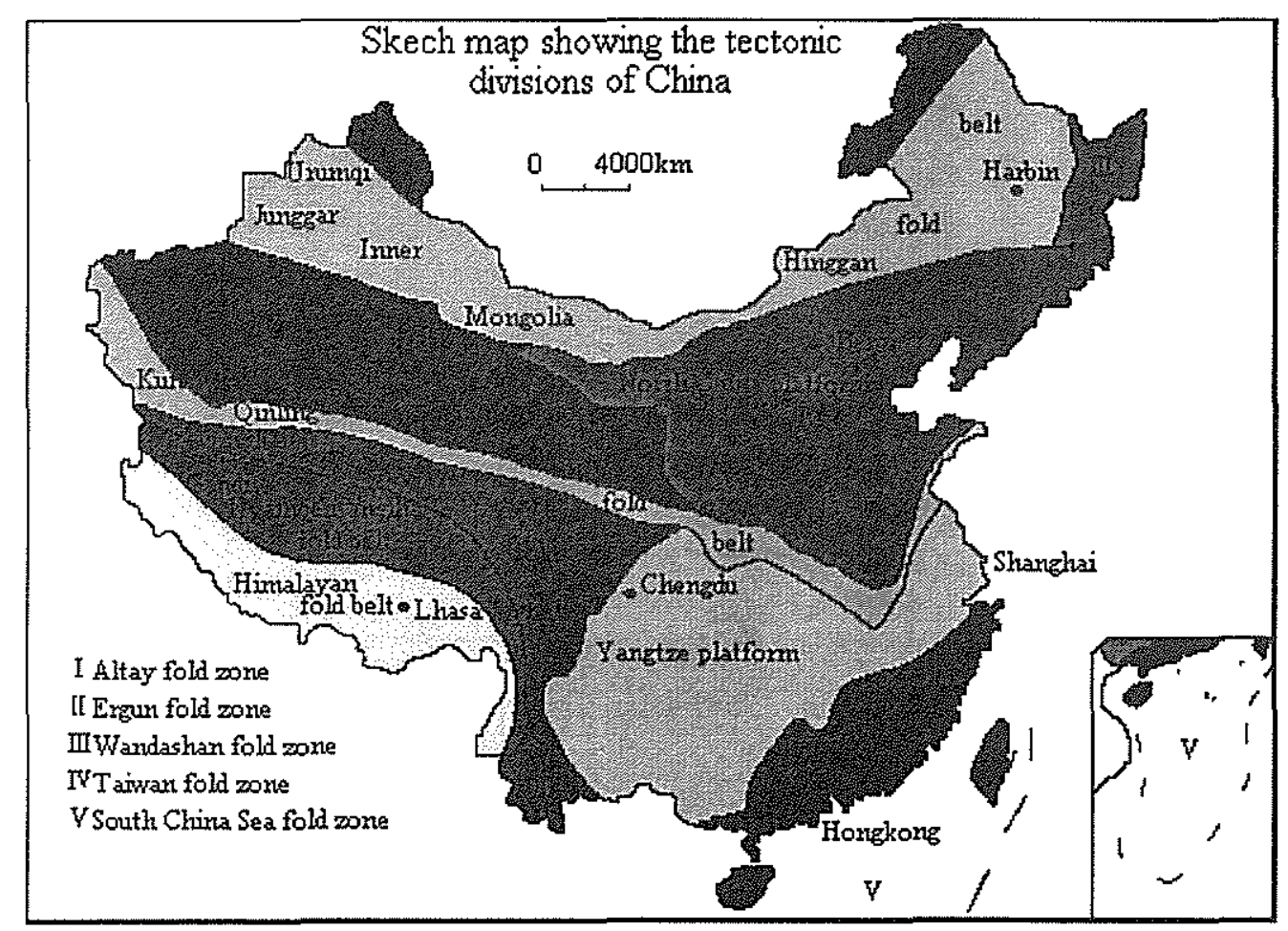

Fig. 1 Tectonic structure sketch of China 


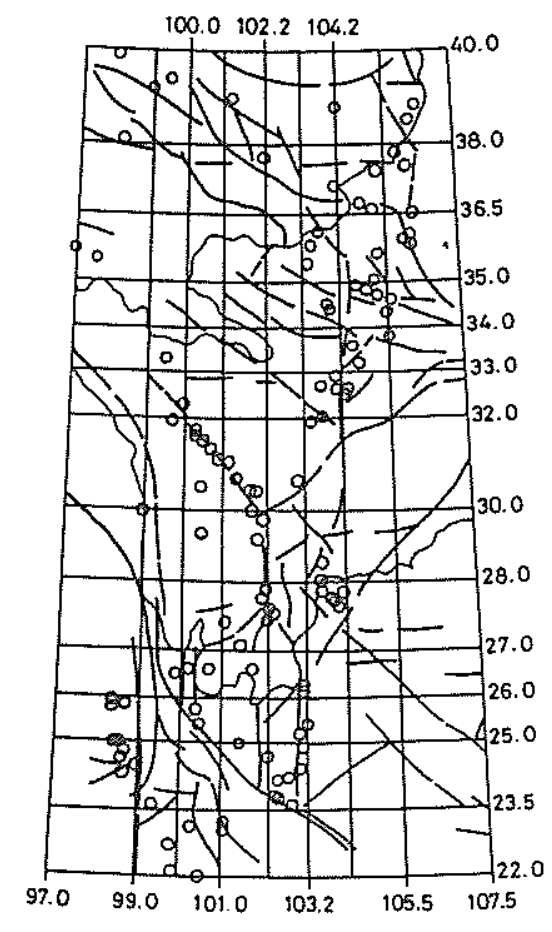

Fig. 2

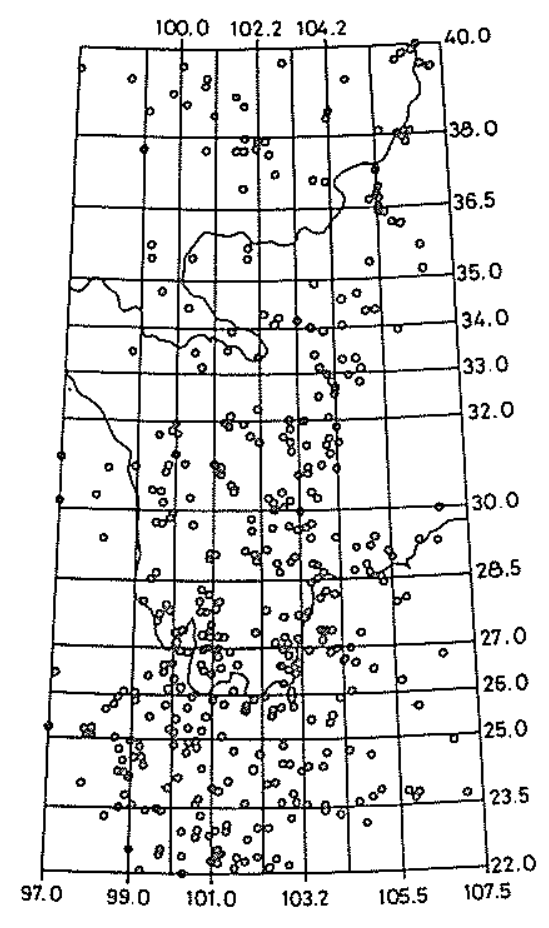

Fig. 3

Active faults and strong earthquake distribution Distribution of regional earthquake epicenters

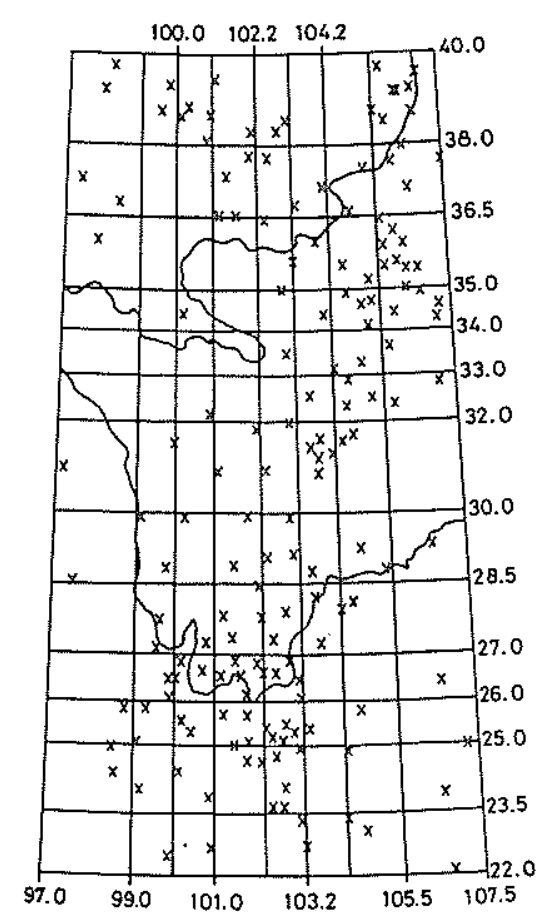

Fig. 4 Distribution of stations 


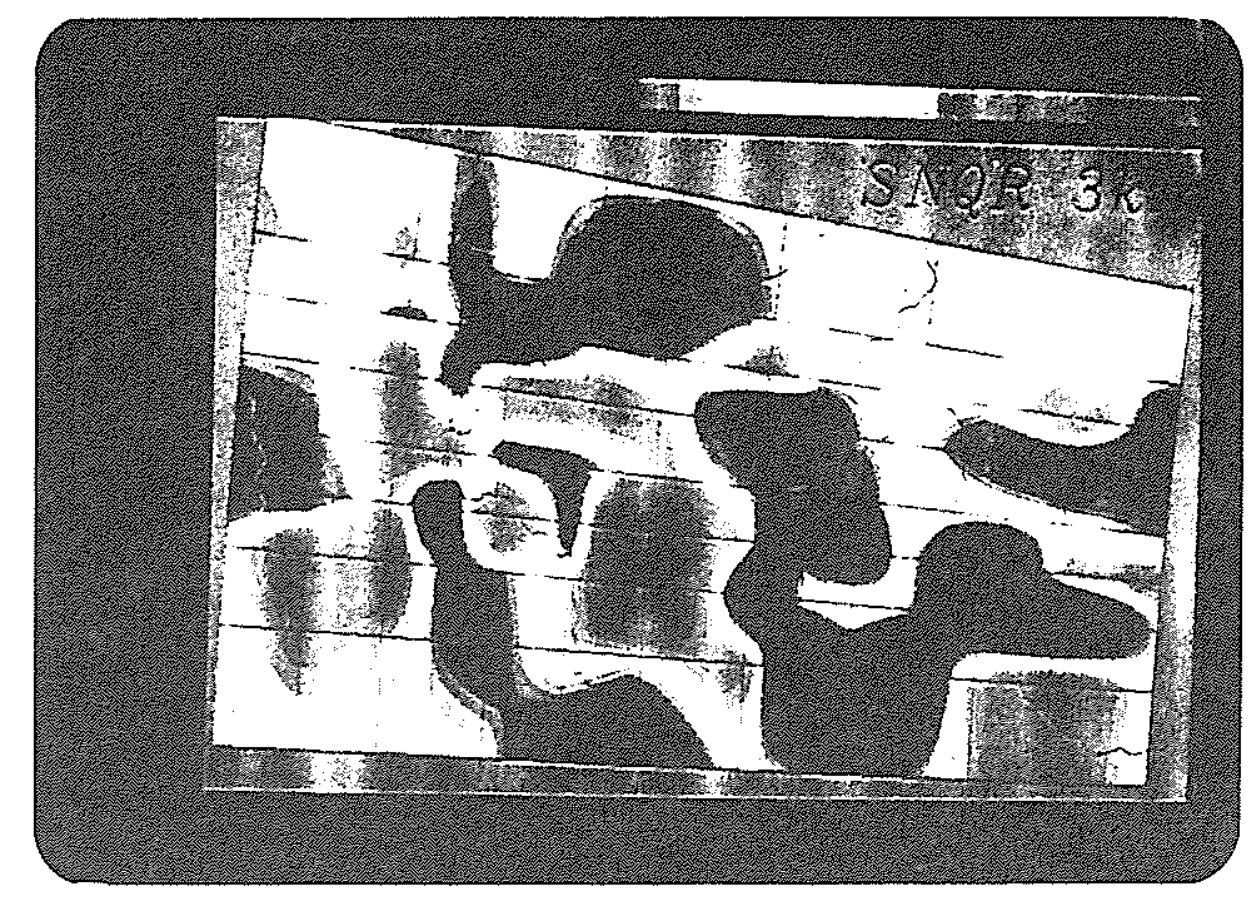

Fig. 5 Velocity image at a depth $3 \mathrm{~km}$

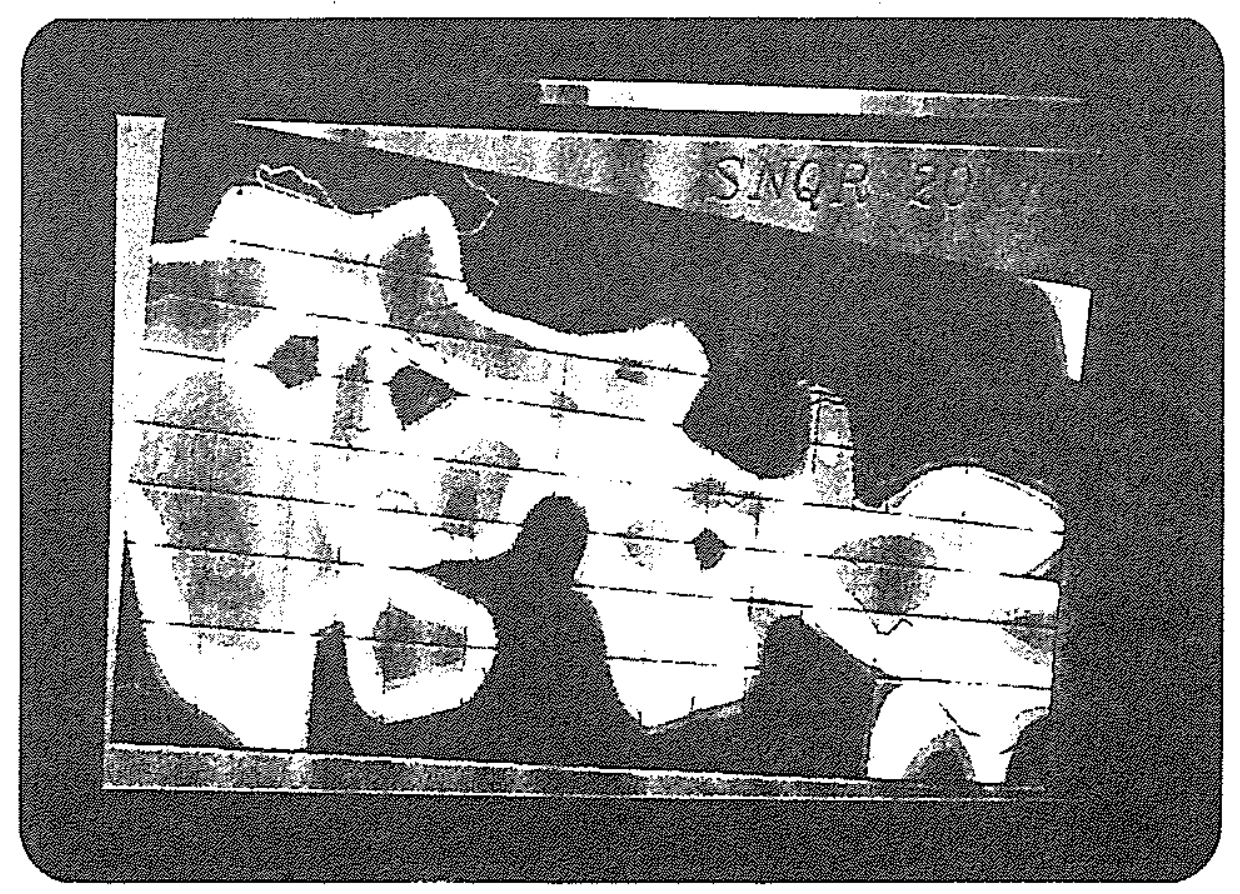

Fig. 6 Velocity image at a depth $20^{+0} \mathrm{~km}$ 


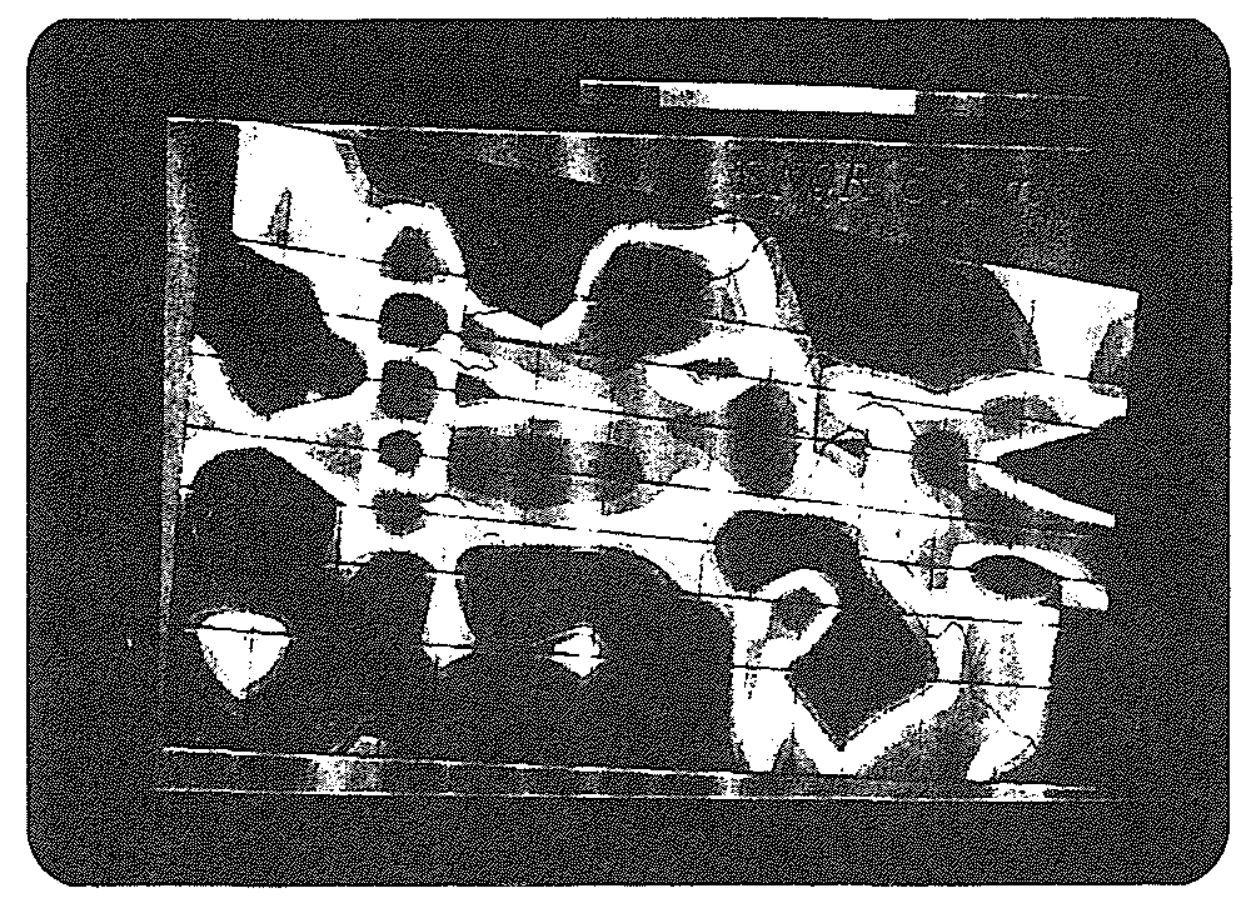

Fig. 7 Velocity image at a depth $50 \mathrm{~km}$

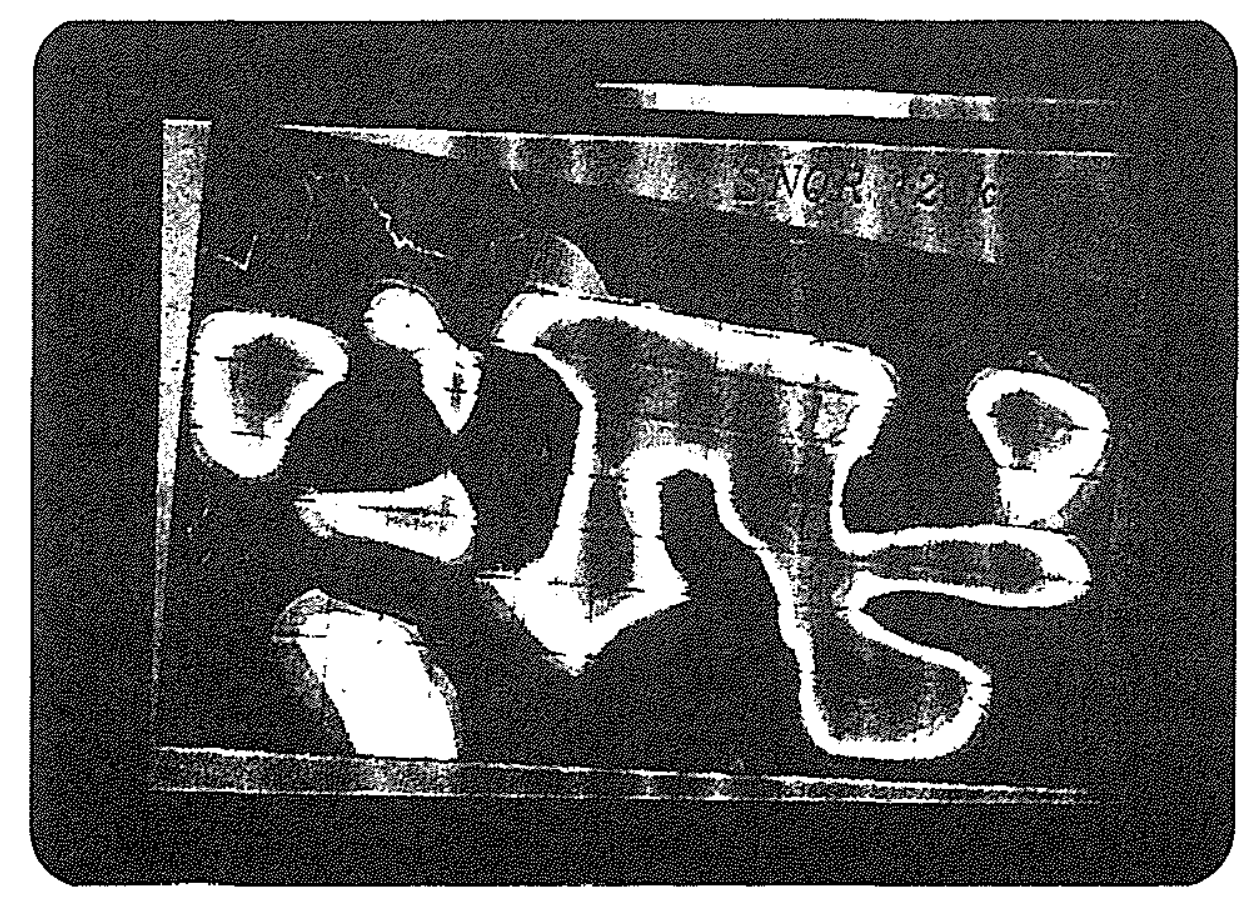

Fig. 8 Velocity image at a depth $120 \mathrm{~km}$ 